\title{
Design and Implementation of Density-Based Traffic Management System
}

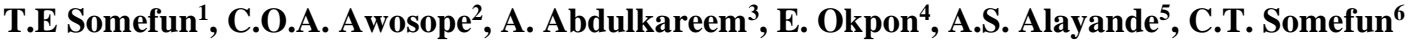 \\ 1,2,3,4Electrical and Information Engineering, Covenant University, Ota, Ogun State, Nigeria. \\ ${ }^{5}$ Electrical and Electronics Engineering, University of Lagos, Akoka Yaba, Lagos Sate, Nigeria. \\ ${ }^{6}$ Computer Science and Engineering, Ladoke Akintola University of Technology, Ogbomosho, Nigeria. \\ ORCID: 0000-0003-1470-5725 (TE Somefun)
}

\begin{abstract}
Traffic congestion and accidents caused by over speeding vehicles have been a major cause for concern in societies due to their negative effects such as stress to commuters, release of more toxic fumes into the atmosphere, accidents and loss of productive hours. The conventional traffic light uses a fixed logic of allocating the same "go time" to lanes at intersections without taking the density of traffic into consideration. This paper offers an approach for handling traffic congestion and over speeding offenders. This system uses infrared sensors as counters to measure the traffic density in each lane. The infrared sensors are also for speed detection with a bluetooth module and bluetooth serial monitor. These sensors are interfaced with the traffic light through a microcontroller. The microcontroller which was used to implement this is the Arduino Mega with the Atmega 2560 chip. The system was developed to alleviate traffic congestion and over-speeding offenders using a case study of an intersection in Uyo, Akwa Ibom State, Nigeria which usually has severe traffic congestion. This intersection consists of Abak Road, Udo Obio and Udo Eduok Streets. This system was tested and all objectives were accomplished. Results obtained from the implementation of the prototype design show that traffic control and speed detection using infrared sensors and Arduino Atmega 2560 give a better performance and the time taken to clear traffic at an intersection reduced significantly with $60 \%$ time saved.
\end{abstract}

Keywords: Traffic; Speed detection; Congestion; Traffic light; Vehicles

\section{INTRODUCTION}

Congestion in urban areas has given rise to the gradual increase in automobiles and vehicles due to the migration of people into urban settlements bringing about different pros and cons. One of such disadvantages is the increase in both human and vehicular movement which additionally leads to high traffic on major roads in a city and therefore prompts activity clog in urban zones. Traffic congestion occurs when the use of road network increases and it is characterized by bad roads in communities and increase in community population. Traffic jam occurs at a point when vehicles completely stop for a period of time. Extreme Congestion occurs mostly during workdays and rush hours (morning and evening).

One of the principal reasons why congestion exists on the roads is due to the increase in the number of vehicles. That is, when the number of vehicles outnumber the capacity of the available infrastructure such as good roads, traffic light and road regulators. As population of urban settlers increases, the need to acquire personal vehicle for transport increases, and traffic congestion increases as the number of vehicles increases combined with an absence of proper infrastructure. The national government and councils neglect to follow up on the approaching danger of increased congestion until it eventually occurs. The roads of the city do not expand in size alongside an inexorably vehicle dependent populace. An example is a single street with one lane on each side before will probably not get developed in ten years after the population has expanded. The authorities frequently neglect to take this into consideration.

The rapid increase in the number of vehicles without a rapid increase in road networks is the main cause of congestion which is a major concern in the society. While it may be difficult to totally tackle congestion, there are a few different ways to control its future rate of increment like: quick response to traffic- blocking incidence and accidents, removing mischances from major roads quickly by utilizing the service of roving vehicles run by Government Traffic Management Centers (GTMC); This is an incredible strategy for diminishing blockage delays, building of more roads in developing regions and improving on urban region road network, and car-sharing service which would reduce the need to own personal vehicles thereby 
reducing the number of cars on the road.

Over the years, traffic congestion has been a cause for concern in the society due to its negative effects like stress to commuters, release of more toxic fumes into the atmosphere and loss of productive hours [1]. While trying to address this issue, diverse researchers have designed and executed certain techniques to help ease traffic in major roads. The first of such technique was actualized by a police officer in Detroit in 1912 and changed in 1920 by another policeman William Port making use of similar standards yet with different modifications. Without question, most traffic lights presently being used today have kept on serving the need for which they were produced. Nonetheless, a new method that will help to combat the issue of traffic congestion largely as automation is a trend which developed countries are imbibing in order to minimize accident and maximize efficiency [2-4]. Traffic light, which can also be called traffic lamps or traffic control signals, are devices that are stationed at strategic location like road intersections to control the flow of traffic. Traffic control signal shows the right of way to users at a particular time by displaying lights of standard color (red, yellow, and green). The red light is a clear caution to "stop" prohibiting any vehicle from moving further while the green light simply means "go". This is set to permit vehicular movement in the denoted direction. The yellow light warns the driver that the signal is about to change to red, it definitely does not mean full- throttle ahead but rather slow down and prepare to stop but most road users fail to adhere to this. This is one of the issues this study seeks to curb.

Installing a traffic light at a crossway should be considered at a strategic location where the presence of the traffic signal will be of high impact rather than create problems. A properly installed and operating traffic signal helps in orderly movement of traffic, thereby reducing the occurrence of possible vehicle clashes. Therefore, before any traffic signal is installed, crash statistics should be analyzed, speed data should be studied, and most importantly road conditions should be examined. For a traffic light to serve its purpose, it must be properly designed, located and maintained regularly.

Currently, traffic lights are set in different lanes with fixed time delays, following a specific cycle while changing from one signal to another making undesirable and unwanted congestion on one lane with alternate lanes stay empty. It is in view of this situation which often times results in the loss of productive time that the density-based traffic management system is proposed.

Hence, mulling over the extremely bustling nature of our roads particularly amid peak hours, there arises a dire need for a more effective traffic management system. One which will reduce congestion in most bustling junctions [5-8].

It is in view of this challenge that this work is carried out to regulate traffic control and monitor speed limit with addition input that is absent in previous design. The method being proposed here is different from the current design that has a fixed time to control and monitor traffic irrespective of the traffic flow. The proposed design would depend basically on the density of each lane by optimizing the "go time" allocated to a lane. This is because it will be a waste of productive time and inappropriate time-sharing formula when a congested lane is allocated the same "go time" with a relatively less busy lane at a junction. The proposed design would provide the quickest possible clearance to congested vehicles in all directions at any junction. The design would reduce the frequent occurrence of accidents resulting from the lack of patience by road users. Furthermore, Over-speeding is one of the main reasons of accidents on our roads will also be curbed. The speed detector incorporated in the proposed system would help onroad police in tracking and detecting traffic defaulters.

\section{REVIEW OF PAST RELATED WORKS}

Traffic control system which is usually stationed at strategic location like road intersections to control the flow of traffic is dated as far back 1868. The history and evolution of traffic control system can be found in [9-14]. Traffic control signal shows the right of way to users at a particular time by displaying lights of standard color (red, yellow, and green). The red light is a clear caution to "stop" prohibiting any vehicle from moving further while the green light simply means "go". This is set to permit vehicular movement in the denoted direction. The yellow light warns the driver that the signal is about to change to red, it definitely does not mean full- throttle ahead but rather slow down and prepare to stop but most road users fail to adhere to this. This is one of the issues this study seeks to curb.

Chinyere et al. (2011) proposed an intelligent system for controlling traffic which was designed by combining Structured Systems Analysis/Design Methodology (SSADM) and the Fuzzy-Logic- based Design Methodology. Two electromagnetic sensors were placed on the road with the second sensor placed 
behind the first sensor. While one sensor counts the number of cars that go past the traffic light, the other counts the number of cars coming from a distance and the difference between the two is evaluated. There is one state for each phase of the traffic light and the state machine controls the sequence of state of the fuzzy traffic controller and a state is skipped if there are no vehicles [5]. In [15], the authors proposed a system which uses a Raspberry pi as its microcontroller to provide the signal timing based on the traffic density i.e., the signal timing changes automatically on sensing the traffic density at the junction. The cameras were placed on top of the signals for a clearer view. The image captured in the signal was first processed then converted into grayscale image. Its threshold was calculated based on the contour which has been drawn in order to calculate the number of vehicles present in the image. After the calculation on the number of vehicles was done, the outcome helped to know which side the density is high based on which signals will be allotted for a particular side. An adaptive traffic light control was proposed in [16] using a wireless sensor network which is classified into three categories namely; the wireless sensor network, the localized traffic flow model policy and the higher level coordination of the traffic lights agents. Wireless sensors were stationed on the lanes going in and out of the intersection. The function of the sensors is to identify and detect car number, speed and some other variables; before sending the information to the nearest Intersection Control Agent who will decide on the flow model of the junction depending on the data from the sensor. Ganiyu et al. (2014) designed a system using Programmable Integrated Circuit (PIC) 16F84A microcontroller, power section, crystal oscillator and light emitting diode (LED). To control traffic effectively, the PIC was implemented via an integrated circuit (IC) programmer using a mikrobasic program which is written in Basic language. The sensing unit used is a pressure switch which detects the weight of vehicle that steps on it and sends a signal to the microcontroller. [17] improved on this work by including surveillance system. Many researches have thought of various models to manage traffic congestion and these models can be grouped under three categories- namely network, application and acquisition. The acquisition layer is made of sensors like infrared (IR), RFID among others while application category includes information collection and analysis. Network category is made of cellular mobile network, Bluetooth, Wi-Fi etc. [18]

\section{MATERIALS AND METHOD}

The system design in this work is a small prototype and it is divided into four units namely; power supply unit, sensor unit, control unit and indication unit.

\section{III.I Power Supply Unit}

The power supply unit is responsible for supplying power needed by the system. The voltage required by the system is $9 \mathrm{~V}$. The power supply consists of the transformer that steps down $220 \mathrm{~V}$ alternating current to $12 \mathrm{~V}$ ac. A bridge rectifier combined with the filtering capacitor was used to convert the alternating current to direct current. A voltage regulator was then deployed to make sure that the voltage is steady at the desired $9 \mathrm{~V}$. The block diagram of regulated power supply unit is displayed in Fig. 1.

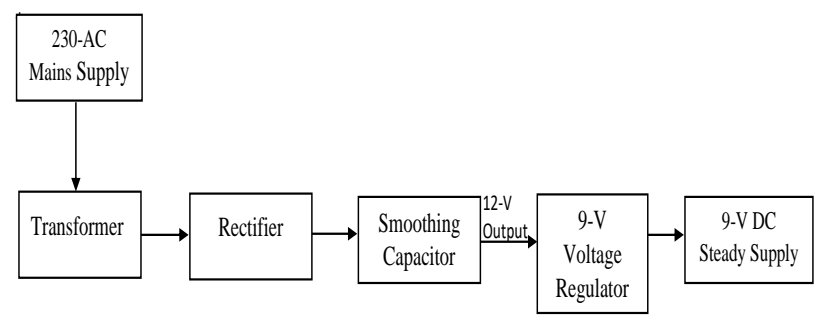

Fig. 1. Block diagram of regulated power supply Sensor Unit

The sensor unit is made up of infrared sensors. The infrared sensor designated for density management detects the density of vehicles on each lane while the infrared sensors designated for speed detection (located a bit far from the traffic light) senses the speed of passing vehicles. It provides input to the controller. The choice of the sensor used was guided by the following criteria:

- Accuracy;

- Cost;

- Range; and

- Resolution - Smallest.

The emitter which is the infrared LED emits infrared radiations which are invisible to the human eye while the photodiode which acts as the receiver detects this radiation sent out by the emitter. Infrared photodiodes can only detect infrared radiation which differentiates them from regular light emitting diodes. Figures 2 and 3 show the basic working principle of the infrared and IR module used in this work respectively.

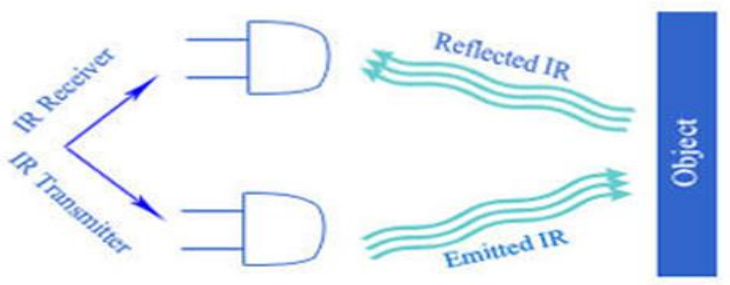

Fig. 2. Infrared basic working principle 


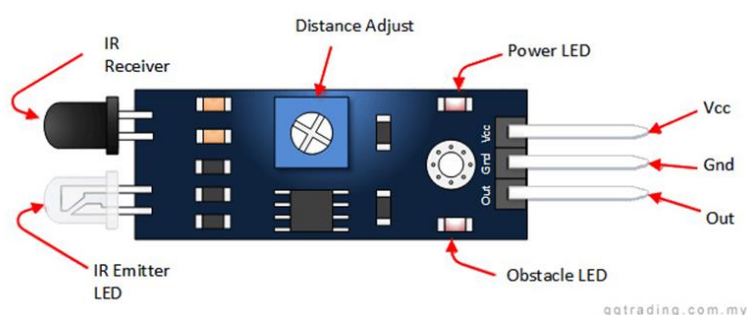

Fig. 3. Infrared basic working principle

\section{III.II Control Unit}

The control unit is a microcontroller-based control system. Fig. 4 gives the pictorial view of the Arduino Mega used in this work. It works by interpreting the input, qualifies it and produces a desired output. Arduino mega 2560 is part of the family of arduino microcontroller boards which is manufactured under the principle of ATmega 2560. It was made with the intent of having a fully self-sufficient microcontroller, consisting of 54 digital input/output pins, 16-MHZ crystal oscillators, power jack, reset button and a USB port/connection so as to be configured by an end user computer. It is powered by a $9-\mathrm{V}$ DC supply. The specification of the Arduino Mega2560 is displayed in Table 1.

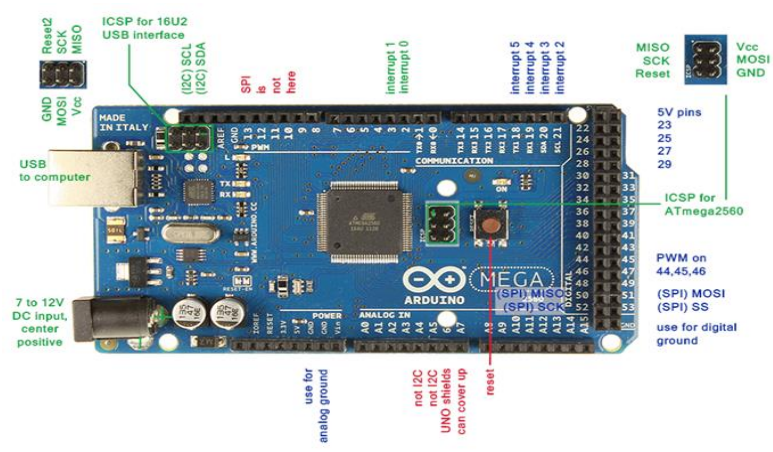

Fig. 4. Arduino Mega

Table 1: Arduino Mega2560 specification

\begin{tabular}{|l|l|}
\hline ATmega2560 & Specification \\
\hline Operating Voltage & $5-\mathrm{V}$ \\
\hline $\begin{array}{l}\text { Recommended } \\
\text { Input Voltage }\end{array}$ & $7-\mathrm{V}$ to $12-\mathrm{V}$ \\
\hline USB Port & Yes \\
\hline DC Power Jack & Yes \\
\hline Digital I/O Pins & 54 \\
\hline $\begin{array}{l}\text { DC current on I/O } \\
\text { Pins }\end{array}$ & $20 \mathrm{~mA}$ \\
\hline Flash memory & 256 \\
\hline SRAM & $8 \mathrm{~KB}$ \\
\hline EEPROM (Clock & $4 \mathrm{~KB}$ \\
\hline $\begin{array}{l}\text { Frequency } \\
\text { Speed) }\end{array}$ & $16 \mathrm{MHz}$ \\
\hline
\end{tabular}

\section{III.III Indicator Unit}

The indicator unit is made up of the bluetooth module /serial interface and also the light emitting diodes (LEDs) which are used to represent the traffic lights.

Wireless data transmission can be easily accomplished with the use of a Bluetooth module. The Bluetooth module has an operating frequency of $2.4 \mathrm{GHz}$ which is among the popular industrial, scientific and medical (ISM) frequency band. The interface used to set this module is a serial interface which is not difficult to use making the development cycle and overall design simplified. This Bluetooth interface is designed strategically to work with android and windows operating system. When the authorized speed on a particular lane is being followed, the microcontroller detects this and no action is taken but when an unauthorized speed is detected by the sensors and a signal is sent to the microcontroller. The microcontroller then sends a signal to the Bluetooth sensor thereby authorizing it to send a code word to the Bluetooth serial monitor telling the authorities that the speed limit has been violated. The specifications of the bluetooth module is shown in Table 2 .

Table 2: Bluetooth module specification

\begin{tabular}{|l|l|}
\hline \multicolumn{1}{|c|}{ Bluetooth Protocol } & \multicolumn{1}{|c|}{$\begin{array}{c}\text { Bluetooth 2.0 + EDR } \\
\text { standard }\end{array}$} \\
\hline USB protocol & USB v1.1/2.0 \\
\hline Operating frequency & $\begin{array}{l}2.4 \mathrm{GHz} \text { ISM frequency } \\
\text { band }\end{array}$ \\
\hline Mode of modulation & $\begin{array}{l}\text { Gauss frequency shift } \\
\text { keying }\end{array}$ \\
\hline Power transmitted & $\leq 4 \mathrm{dBm}$, second stage \\
\hline Sensitivity & $\begin{array}{l}\leq-84 \mathrm{dBm} \text { at } 0.1 \% \text { Bit Error } \\
\text { Rate }\end{array}$ \\
\hline Transmission speed & $\begin{array}{l}2.1 \mathrm{Mbps} \text { (Max)/ 160 kbps ( } \\
\text { Asynchronous); 1Mbps/ } \\
1 \mathrm{Mbps} \text { (synchronous) }\end{array}$ \\
\hline Safety feature & Authentication\& encryption \\
\hline Supported configuration & $\begin{array}{l}\text { Bluetooth serial port (major } \\
\text { and minor) }\end{array}$ \\
\hline Voltage supplied & $+3.3 \mathrm{VDC} 50 \mathrm{~mA}$ \\
\hline Operating temperature & -20 to $55^{\circ} \mathrm{C}$ \\
\hline Size & $36.5^{*} 16 \mathrm{~mm}$ \\
\hline Weight & $4 \mathrm{~g}$ \\
\hline
\end{tabular}

The block diagram, circuit diagram and flow chart of the entire system are as shown in Figures 5, 6 and 7.

As seen from the flow chart, the system starts and so does the microcontroller. All the stop flags are set. This means that all the traffic light indicators on the four lanes of the road intersection show a RED light 
which stops all traffic at the beginning of its operation. At "A" the infrared sensors on lanes 1 and 3 detect density on the lanes and compare them and give priority to the lane with more density then infrared on lanes 2 and 4 also detects density and the process continues. If there are no vehicles indicating density on any lane, the traffic light for those lanes remain on "RED". A comprehensive flow chart for the proposed method is given in the supplementary file.

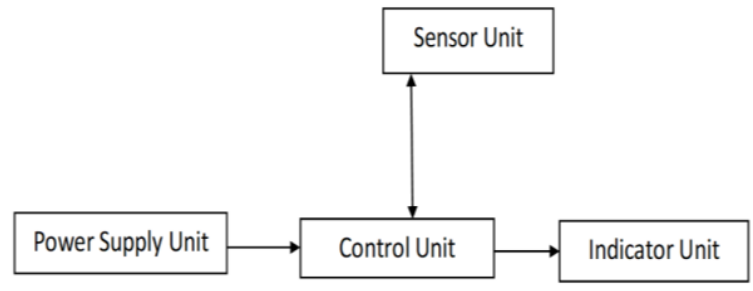

Fig. 5. Block diagram of density-based traffic management system

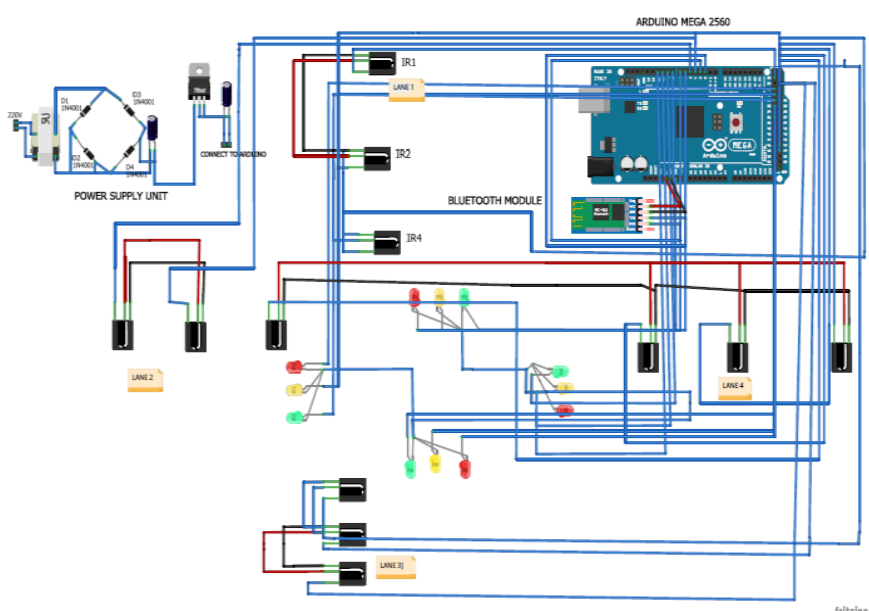

Fig. 6. Circuit Diagram
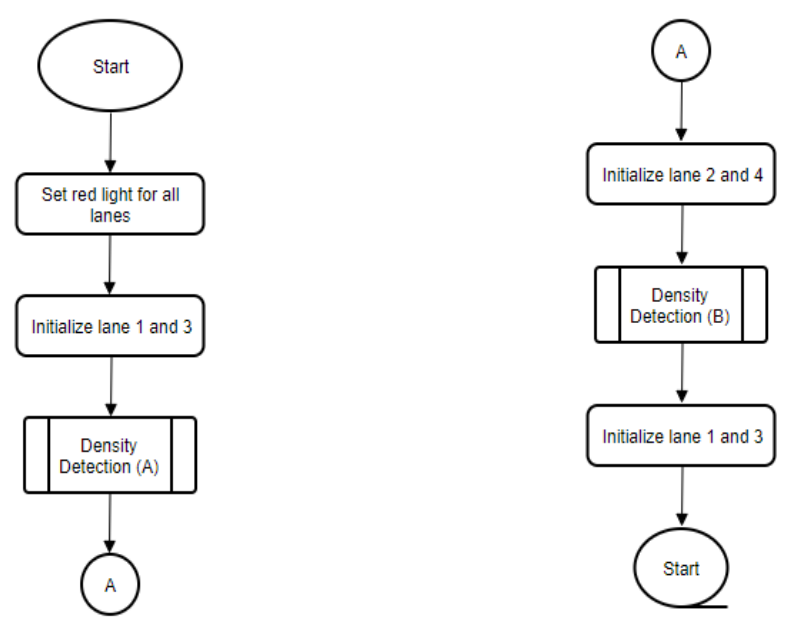

Fig. 7. Flow Chart of Density-Based Traffic Management System

\section{1) Infrared (IR) Sensor used as a density management component}

The IR sensor in this system is used as a counter and each input from the sensor causes a digital signal output that triggers the LED's (traffic lights). A single infrared sensor is used to act as a counter for multiple vehicles coming towards the traffic light. A time is set for the number of vehicles that pass per interval, due to the small prototype design, if the IR sensor detects one vehicle per interval, a standard programmed time of six seconds is allocated for the green LED (Green light) to stay on. If the IR sensor detects two vehicles per interval, a standard programmed time of nine seconds is allocated for the green LED (Green light) to stay on. If the IR sensor detects three or more vehicles per interval, a standard programmed time of fifteen seconds is allocated for the green LED (Green light) to stay on. This explains the working principle of the traffic density management system of this system.

\section{2) Infrared Sensor used as a speed detector}

Two independent IR sensors are used to act as a speed detection system for this work. The two IR sensors are programmed and linked to each other: the first IR sensor is labelled 1 and the second is labelled 2 . When a signal is sensed by IR 1, a timer is started automatically until a second signal is detected by the second IR sensor labelled 2; the moment that happens, the time difference between the first signal detected and the second signal is converted to speed as follows:

The distance between the two IR sensors used for speed detection at each lane is $6 \mathrm{~cm}$ i.e. $0.06 \mathrm{~m}(1 \mathrm{~m}=100 \mathrm{~cm})$. The time recorded is in millisecond and $(1$ millisecond $=$ $10^{-3}$ second).

Therefore,

$$
\begin{aligned}
\text { Speed } & =\frac{0.06}{1 \times 10^{-3}} \\
& =60 \mathrm{~m} / \mathrm{s}
\end{aligned}
$$

When converting from $\mathrm{m} / \mathrm{s}$ to $\mathrm{km} / \mathrm{h}$, we have

$$
\begin{aligned}
& \frac{\mathrm{m}}{\mathrm{s}}=\frac{\frac{1}{1000} \times \mathrm{km}}{\frac{1}{3600} \times \mathrm{h}} \\
& \frac{\mathrm{m}}{\mathrm{s}}=\frac{3600 \times \mathrm{km}}{1000 \times \mathrm{h}} \\
& \frac{\mathrm{m}}{\mathrm{s}}=\frac{18 \times \mathrm{km}}{5 \times \mathrm{h}}
\end{aligned}
$$

Hence, to convert from $\mathrm{m} / \mathrm{s}$ to $\mathrm{km} / \mathrm{h}$, the answer is 
multiplied by $18 / 5$ or 3.6

Therefore, $60 \mathrm{~m} / \mathrm{sec}$

$$
\begin{gathered}
=60 \times \frac{18}{5} \mathrm{~km} / \mathrm{hr} \\
=216 \mathrm{~km} / \mathrm{hr}
\end{gathered}
$$

If two IR sensors are to be placed $5 \mathrm{~cm}$ apart, then $0.05 \mathrm{~m}$ is chosen instead of 0.06 . The same is done for other distance between two IR sensors.

Once the conversion of time to speed has been done, a threshold speed which has been preprogrammed into the microcontroller comes into play by comparing the threshold speed with the converted speed and if the converted speed is lower than threshold speed, then no signal is sent to the microcontroller meaning that the car is moving at the authorized speed but if the converted speed is higher than the threshold speed, then a signal is sent from the microcontroller to the bluetooth serial monitor (android or windows application) through the bluetooth module. The flow chat for the speed detection is given in Figure 8.

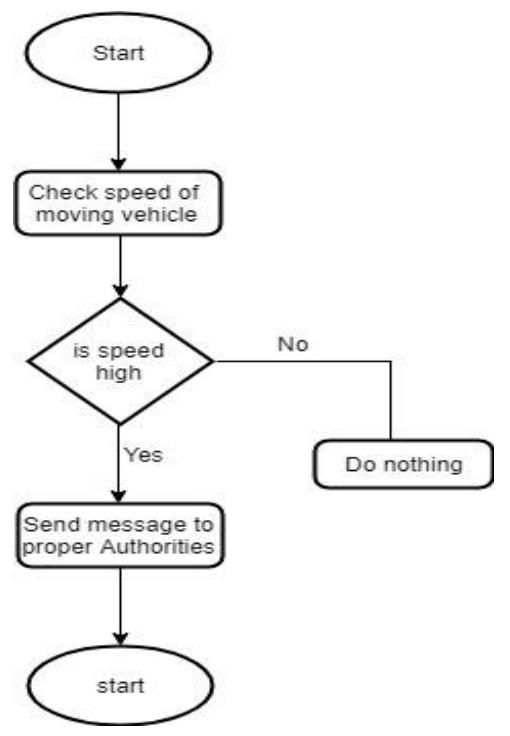

Fig. 8. Flow Chart of Speed Detection System

To ensure that the system works properly, various components used in the design of the work were tested. During construction, continuity test was carried out on the circuit at different stages. In the power supply unit, the transformer, the bridge rectifier and other components were tested to ensure the desired voltage level was obtained.

The application used in this work was programmed to connect to the Bluetooth interface of the work and also the application was built to run on both android and windows operating system, The application was tested by connecting it to the bluetooth device on the hardware which allows the application to receive all speed default notifications.

For the software implementation of this work, the Arduino Atmega 2560 Microcontroller was programmed at the Arduino Integrated Development Environment (IDE) which was installed on a personal computer. The codes for the Arduino were written in $\mathrm{C}$ language. After cross checking the written program, the codes were compiled and uploaded to the Arduino board using its USB port.

\section{RESULT}

The location noted for traffic jam and its road layout must be taken into consideration for any traffic issue to be resolved. This work can be implemented in other places but the location considered for the implementation of this work is the cross road made of Abak Road, Udobio Street and Udo Eduok Street in Akwaibom State, Nigeria. Abak Road is a major lane while Udobio Street and Udo Eduok Street are quite small and they sometimes have low car density.

This location was chosen due to the constant traffic jam. A conventional traffic light is installed at this location but it is obviously not efficient. During traffic control, smaller Streets like Udo Eduok and Udobio were allocated "equal go time" with the Abak Road even when there was no traffic on Udo Eduok and Udobio Streets. This usually caused undue traffic jam on Abak Road and as a result, time wasting leading to the road users trying to outrun traffic lights or over speeding at ready time (yellow light) thereby causing accidents many times. A representation of the considered location is represented in Fig. 9, while the 2-D view is shown in Fig. 10.

Figure 11 shows the output of high speed detected on the lanes, through the Bluetooth terminal. This can be used by the local police who are always on standby to enforce speed limit or sanction offenders appropriately.

Table 3 shows a hypothetical situation with empirical values for the chosen location for the implementation in order to ascertain the effectiveness of the proposed method. 


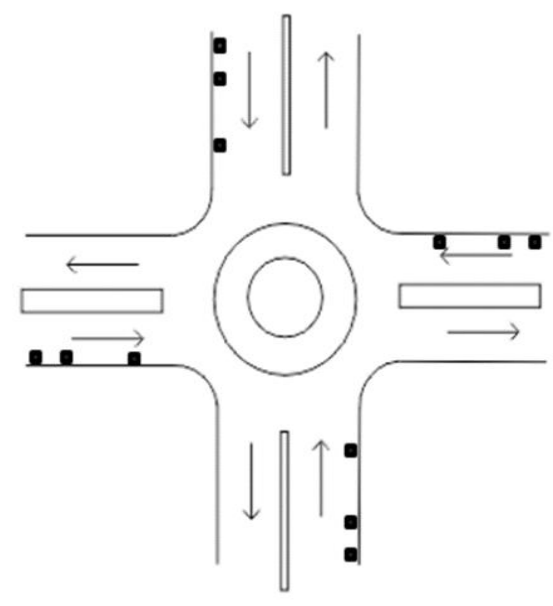

Fig. 9. Schematic Diagram of Proposed Road Layout for

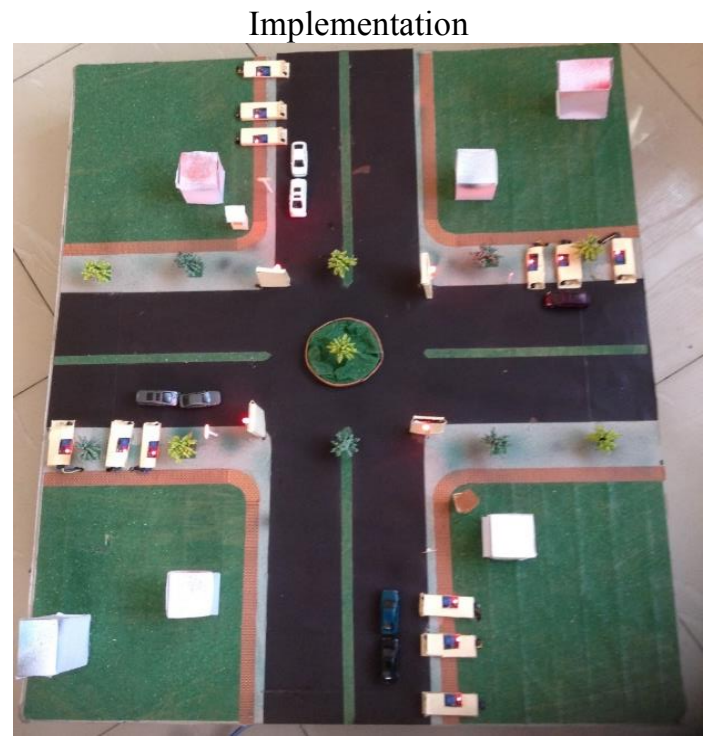

Fig. 10. Pictorial View of Constructed Prototype

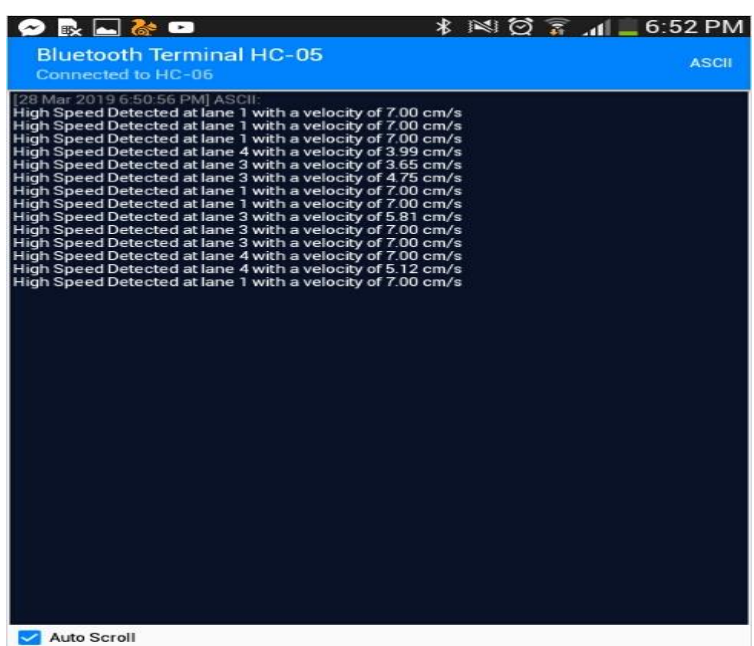

Fig. 11. High speed detection shown through Bluetooth terminal
Table 3: Table showing number of cars on each lane

\begin{tabular}{|l|l|l|l|l|}
\hline Lane & 1 & 2 & 3 & 4 \\
\hline $\begin{array}{l}\text { Number of } \\
\text { cars by the } \\
\text { traffic light }\end{array}$ & 2 & 1 & 5 & 2 \\
\hline
\end{tabular}

\section{DISCUSSION}

For the operation of the proposed design, the adjacent lanes work together. If low traffic is detected on one lane and medium traffic on the adjacent lanes, the "go time" for both lanes will be according to the highest traffic lane between both of them. Therefore, in the case stated above, those lanes will be allocated 9 seconds "go time" which is for medium traffic.

Based on the configuration of the available traffic light system, which allocate the same "go time" to each lane, assuming the preprogrammed "go time" for each lane is 15 seconds and it takes approximately 1 second for each vehicle to move past the traffic light, then, it would take 60 seconds for all the lanes to be cleared. Using the proposed real-time system in this work for this same scenario, it would take 24 seconds for all the lanes to be cleared which yields $60 \%$ time saved.

Applying this model to real life situation will go along way to reduce traffic to bearable minimum time interval and caution all drivers to avoid exceeding speed limit which will also eradicate accidents due to over-speeding at cross roads.

\section{CONCLUSION}

The implementation of a smart traffic light system with a speed detection system will, to a very large extent, reduce road accidents caused by over-speeding vehicles and also lessen traffic congestion in our society. Though the work has achieved its main objectives of smart traffic control and high-speed detection, there is the need for more research and improvements. The following recommendations are hereby suggested:

- A system where WIFI technology should be used as it has a wider range and more functionalities than bluetooth technology;

- The system should be powered by a solar system to promote green energy operations and reduce consumption of electricity from the grid; and

- A surveillance system should be installed to capture the vehicles going beyond the stated speed limit. 


\section{ACKNOWLEDGEMENT}

The authors wish to appreciate Covenant University for her financial support.

\section{REFERENCES}

[1] C. Uzondu, S. Jamson, and F. Lai, "Exploratory study involving observation of traffic behaviour and conflicts in Nigeria using the Traffic Conflict Technique," Safety Science, vol. 110, pp. 273-284, 2018/12/01/ 2018.

[2] M. Pau, E. Patti, L. Barbierato, A. Estebsari, E. Pons, F. Ponci, et al., "A cloud-based smart metering infrastructure for distribution grid services and automation," Sustainable Energy, Grids and Networks.

[3] S. Uzairue, J. Ighalo, V. O. Matthews, F. Nwukor, and S. I. Popoola, "IoT-Enabled Alcohol Detection System for Road Transportation Safety in Smart City," in International Conference on Computational Science and Its Applications, 2018, pp. 695-704.

[4] F. E. Idachaba, J. O. Olowoleni, A. E. Ibhaze, and O. O. Oni, "IoT Enabled Real-Time Fishpond Management System," 2017.

[5] O. U. Chinyere, O. O. Francisca, and O. E. Amano, "Design and simulation of an intelligent traffic control system," International journal of advances in engineering \& technology, vol. 1, p. 47, 2011.

[6] R. Ganiyu, O. Arulogun, and O. Okediran, "Development Of A Microcontroller-Based Traffic Light System For Road Intersection Control," International journal of scientific \& technology research, vol. 3, pp. 209-212, 2014.

[7] G. Balan and S. Luke, "History-based traffic control," in Proceedings of the fifth international joint conference on Autonomous agents and multiagent systems, 2006, pp. 616-621.

[8] N. B. Soni and J. Saraswat, "A review of IoT devices for traffic management system," in 2017 International Conference on Intelligent Sustainable Systems (ICISS), 2017, pp. 1052-1055.

[9] L. Day and I. McNeil, Biographical dictionary of the history of technology: Routledge, 2002.

[10] R. L. Gordon, W. Tighe, and I. Siemens, "Traffic control systems handbook," United States. Federal Highway Administration. Office of Transportation Management2005.

[11] P. Pretorius, S. Van As, and R. Troutbeck, "Issues with the operational analysis of urban intersections," SATC 2004, 2004.

[12] J. Ben-edigbe and I. B. M. Ibrahim, "Empirical Delays from Actuated and Optimised Static Signal Settings Compared," 2010.

[13] H. J. van Zuylen and F. Viti, "Delay at controlled intersections: the old theory revised," in Intelligent Transportation Systems Conference, ITSC, 2006.
[14] M. J. Panaggio, B. J. Ottino-Löffler, P. Hu, and D. M. Abrams, "Symmetry breaking in optimal timing of traffic signals on an idealized two-way street," Physical Review E, vol. 88, p. 032801, 2013.

[15] K. Vidhya and A. B. Banu, "Density based traffic signal system," International Journal of Innovative Research in Science, Engineering and Technology, vol. 3, pp. 2218-2222, 2014.

[16] M. Tubaishat, Y. Shang, and H. Shi, "Adaptive traffic light control with wireless sensor networks," in Consumer Communications and Networking Conference, 2007. CCNC 2007. 4th IEEE, 2007, pp. 187-191.

[17] Y. Udoakah and I. Okure, "Design and implementation of a density-based traffic light control with surveillance system," Nigerian Journal of Technology, vol. 36, pp. 1239-1248, 2017.

[18] J. Sherly and D. Somasundareswari, "Internet of things based smart transportation systems," International Research Journal of Engineering and Technology, vol. 2, pp. 1207-1210, 2015. 